\title{
(Towards) Spin-polarized scanning tunneling microscopy of NiFe films on a chromium(001) single crystal: Growth and electronic structure of Permalloy
}

\author{
M. Dreyer and J. Lee \\ Department of Elec. and Comp. Eng., University of Maryland, College Park, Maryland 20742 \\ and Laboratory for Physical Sciences, University of Maryland, 8050 Greenmeade Drive, College Park, \\ Maryland 20742 \\ C. Krafft \\ Laboratory for Physical Sciences, University of Maryland, 8050 Greenmeade Drive, College Park, \\ Maryland 20742 \\ R. Gomez \\ Department of Elec. and Comp. Eng., University of Maryland, College Park, Maryland 20742 \\ and Laboratory for Physical Sciences, University of Maryland, 8050 Greenmeade Drive, College Park, \\ Maryland 20742
}

(Presented on 9 November 2004; published online 10 May 2005)

\begin{abstract}
This paper shows spin-polarized tunneling on the terraces of antiferromagnetic chromium(001). Details on the morphological and chemical composition are shown as a function of various stages of surface preparation, and protocols for obtaining $\mathrm{Cr}(001)$ that reveal spin-polarized contrast are reported. On the clean crystal the chromium surface state at the Fermi level could be observed. The spin splitting of the state was measured by using an iron coated tungsten tip. A spin polarization of $10 \%$ was observed. The properties of $\mathrm{NiFe}$ grown on $\mathrm{Cr}(001)$ measured using nonpolarized tunneling spectroscopy are also reported. Monolayer thick permalloy grows in Volmer-Weber mode, exhibiting pyramidal islands oriented along the chromium $\langle 110\rangle$ direction. Spectroscopic data reveal that $\mathrm{NiFe}$ alloying is preserved. (c) 2005 American Institute of Physics.
\end{abstract}

[DOI: $10.1063 / 1.1854420]$

\section{INTRODUCTION}

Spin-polarized scanning tunneling microscopy (SPSTM, for a recent review see Ref. 1), while potentially powerful, is a very subtle technique to observe the magnetic structure of a material down to the atomic level. It is extremely sensitive to the exact condition of the tip and sample, and the results are often convoluted with nonmagnetic influences on the electronic structure, such as step edges or submonolayer contaminations. Therefore, the SPSTM experiments are conducted under ultrahigh vacuum conditions and on wellcharacterized substrate surfaces. An ideal substrate for studying NiFe films is the (001) surface of single crystal chromium as it offers several important benefits. First, $\mathrm{Cr}(001)$ is a topographic antiferromagnet, i.e., the magnetization lies within the surface plane and changes direction by $180^{\circ}$ between adjacent terraces separated by a monoatomic step. This provides an unambiguous magnetic background structure to immediately test the magnetic tips. Spinpolarized tips, for instance, should reveal alternating bright and dark contrast as they scan adjacent monolayer step terraces. ${ }^{2}$ Second, chromium pins the magnetization of the submonolayer NiFe films (similar to Ref. 3), so that even ferromagnetically coated tips can be used for imaging. More importantly, the coupling the ultrathin $\mathrm{NiFe}$ film with the chromium substrate gives a predictable magnetic orientation of the NiFe layer. Thus the interpretation of the data, especially when comparing spectroscopic data derived from spin polarized and nonpolarized tips, is greatly simplified.
Despite the ideal qualities of $\mathrm{Cr}(001)$, it is not straightforward to prepare the surface with a sufficiently low impurity level and high crystalline order to make it suitable for spin-polarized STM. Hence, two of the pragmatic issues are the level of surface condition that would allow spin polarized STM investigations to be carried out and to understand the morphological and spectroscopic behavior of submonolayer $\mathrm{NiFe}$ films grown on this substrate. Additionally, it is instructive to understand the spectroscopy of $\mathrm{NiFe}$ on $\mathrm{Cr}(001)$ using nonpolarized tips to be able to discriminate between spinpolarized effects and nonpolarized variations in the local conductance. In this experiment, we seek to address these issues by providing detailed protocols for the preparation of suitable $\mathrm{Cr}(100)$ surfaces that exhibit spin-polarized tunneling, as well as to spectroscopically characterize NiFe films on $\mathrm{Cr}(100)$.

\section{EXPERIMENT}

Our experiments were performed inside a two chamber ultrahigh vacuum (UHV) system at a base pressure $p_{0}<8$ $\times 10^{-11}$ mbar. One of the chambers was equipped with an ISE 5 ion gun, two FOCUS electron beam evaporators preloaded with $\mathrm{Ni}_{80} \mathrm{Fe}_{20}$ and iron, respectively. The sample heaters were integrated with the sample holders, along with a thermocouple to precisely measure the temperatures. The second chamber was connected to the first by a valve and contained a combined LEED/AES (LEED-low-energy electron diffraction; AES-Auger electron spectroscopy) 


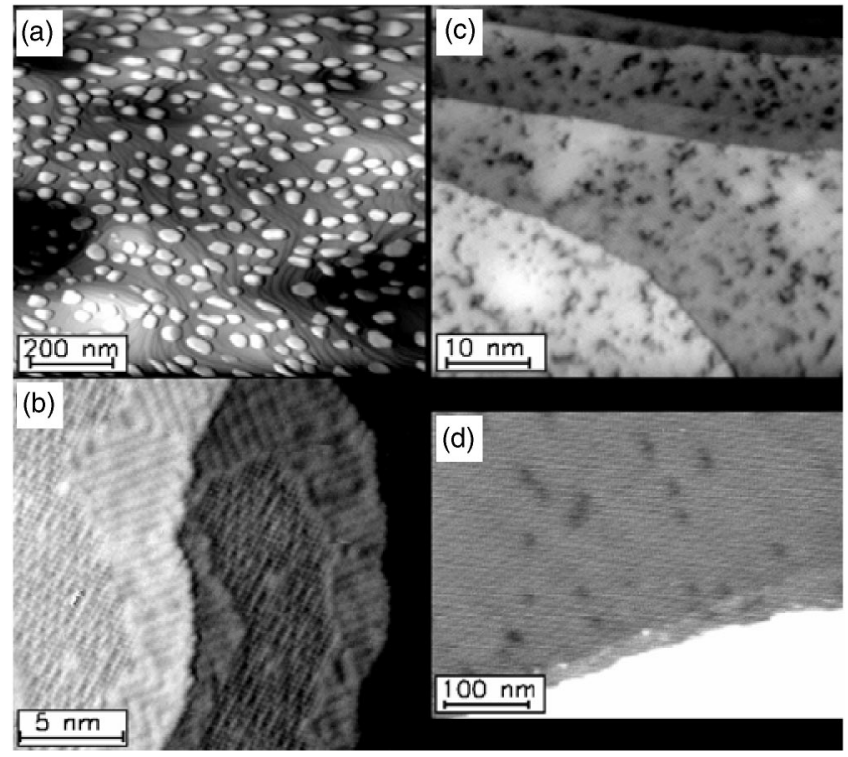

FIG. 1. Several stages of the preparation of the chromium crystal showing (a) oxygen contamination, (b) nitrogen contamination $[c(2 \times 2)$ and $p(2$ $\times 1)$ phase], (c) under annealed $\mathrm{Cr}$, and (d) clean chromium.

system as well as an Omicron room temperature AFM/STM. The STM tips were made from tungsten wire by electrochemical etching using a $\mathrm{KOH}$ solution. It was heated by electron beam bombardment in UHV, while keeping the temperature high enough to remove oxides and other contaminants but without melting the apex.

\section{RESULTS AND DISCUSSION}

The series of STM images in Fig. 1 shows the detailed morphological evolution of $\mathrm{Cr}(001)$ at various stages of the preparation. Figure 1(a) represents the surface after several cycles of sputtering to remove the native oxide and subsequent heating to anneal the surface. We discovered that the use high energies for the argon ions in a range of 1.5-2 keV were optimum in removing large amounts of oxide with a reasonable surface damage. In this specific case, the last step involved sputtering for $120 \mathrm{~min}$, followed by heating to $550{ }^{\circ} \mathrm{C}$ for $270 \mathrm{~s}$. The prominent features are beadlike structures decorating a relatively flat underlayer (similar to Ref. 4). Auger electron spectroscopy showed a significant peak at the oxygen KLL electron energy, which strongly suggests the presence of residual amounts of chromium oxide. The oxide peak was completely removed after a few more cycles of sputtering and heating. The removal of oxygen allowed atomically resolved images of the $\mathrm{Cr}$ surface. Figure 1(b) shows atomic corrugations that form different surface reconstructions. This is due to nitrogen that has been dissolved in the bulk and has migrated towards the surface during the annealing step. As described in Ref. 5, N takes up interstitial sites at the surface leading to various structures, two of which are shown in Fig. 1(b). The image also shows the orientation of the $\mathrm{Cr}$ crystal. The (100) axes was rotated about $25^{\circ}$ counterclockwise with respect to the vertical axes. This was confirmed by a similar value of $30^{\circ}$ derived from LEED measurements. The slight discrepancy can be explained by a misalignment of the camera used to record the
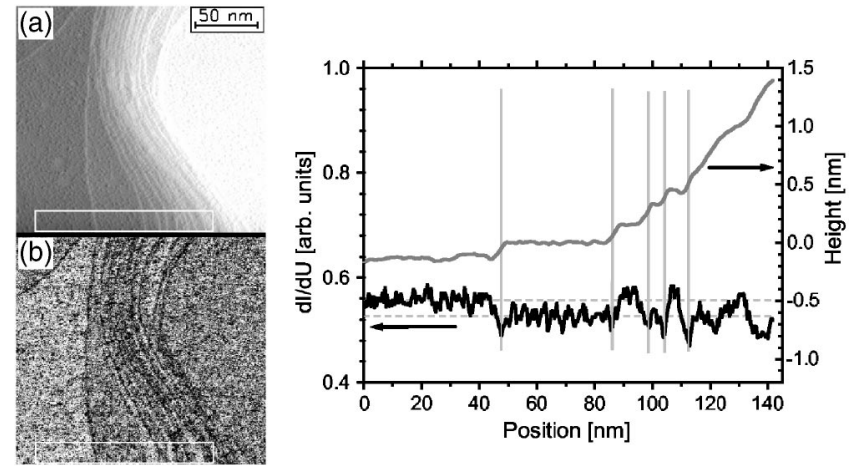

FIG. 2. Spin-polarized tunneling on $\mathrm{Cr}(001)$. The chromium surface (a) shows monatomic steps which are also reflected as a contrast change in the $d I / d U$ map, (b) as emphasized by the cross section $\left(U_{\text {bias }}=-250 \mathrm{mV}\right.$, $U_{\text {mod }}=50 \mathrm{mV}$ ).

LEED images as well as the uncertainty in the orientation of the scan piezo with respect to the sample holder. The nitrogen contamination was clearly visible in spectroscopic data which reveal a peak in the $d I / d U$ maps at an energy of $-0.2 \mathrm{eV}$ - easily distinguishable from the $\mathrm{Cr}$ surface state at the Fermi level. Following the procedure of others, we avoided the $\mathrm{N}$ segregation problem by "flash" annealing of the sample after low energy $(600-800 \mathrm{eV})$ sputtering. In our case, however, this leads to a structure as shown in Fig. 1(c), which despite its roughness is relatively free of nitrogen as evident from the disappearance of the $\mathrm{N}$ surface state at $-0.2 \mathrm{eV}$. After heating the sample to $600{ }^{\circ} \mathrm{C}$ for $15 \mathrm{~min}$ we obtained a flat surface with a low defect/contamination density [Fig. 1(d)].

We discovered that the contrast due to spin-polarized tunneling can be observed even on less than optimized samples, as long as the crystallographic terraces are free of contamination and the tips are properly coated with spinpolarized ferromagnetic material. Following Ref. 1 after cleaning the tip was coated with nominally $11 \mathrm{~nm}$ of Fe according to the calibration of the evaporator. The actual film thickness at the apex might be well below this estimate. The tip was then annealed at $285{ }^{\circ} \mathrm{C}$ for $20 \mathrm{~min}$. An example of SPSTM is shown in Fig. 2 where the surface condition is closest to the one displayed in Fig. 1(d). Despite the roughness of the terraces, it is evident from the STM image that the surface comprises several monatomic steps. As an aide to the eye, the profile of a small rectangular segment is drawn in the accompanying plot. Starting from the left, the profile shows two large terraces of more that $50 \mathrm{~nm}$ in width, followed by a series of closely spaced terraces. The terraces are separated by $1.5 \AA$ high steps. This height matches half the height of a unit cell of $\mathrm{Cr}$. The lower image is the conductance or $d I / d U$ map of the surface at $U_{\text {bias }}=-0.25 \mathrm{~V}$, and was obtained concurrently with the topographic image. It contains the information on spin polarization. Note with care that most of the steps coincide with a contrast change in the $d I / d U$ map [Fig. 2(b)]. The first terrace on the left, for example, has a brighter contrast than the adjacent terrace. This is a consequence of the antiferromagnetic character of the $\mathrm{Cr}$ crystal. The contrast change is also evident in the cross section [Fig. 2(c)], where the locations of the topographic steps 

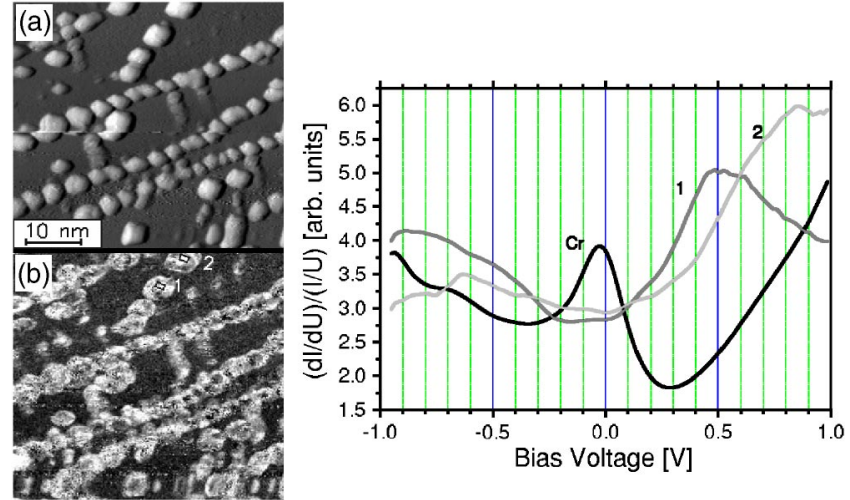

FIG. 3. Growth and electronic structure of $\mathrm{Ni}_{80} \mathrm{Fe}_{20}$ on $\mathrm{Cr}(001)$. (a) shows the topography. The NiFe islands are up to $1.2 \mathrm{~nm}$ in height. (b) shows a $d I / d U$ map at a bias voltage of $550 \mathrm{mV}$. The NiFe islands reveal individual structures against the homogeneous background of Cr. The plot shows normalized spectra of $\mathrm{Cr}$ and the islands marked by "1" and "2" in (b).

are drawn. Indeed, the line profile of the conductance map reveal alternating bright and dark contrast as each monatomic step is crossed. The dark contrast marking the step edge is most likely a scan artifact. By comparing the bright and dark contrast, we find a value of the spin polarization of $P=10 \%$. This is less than the value of $P=20 \%$ previously reported by others. ${ }^{2}$ We attribute this discrepancy as due to the less than optimal magnetic/spin structure of the tip as well as a possible misalignment of the tip magnetization of $60^{\circ}$ with respect to the $\mathrm{Cr}$ magnetic moments.

Finally, we tackle the deposition of $\mathrm{NiFe}$ on the clean $\mathrm{Cr}(001)$ crystal. We evaporated $\mathrm{NiFe}$ at a rate of about 1 monolayer per min. A sample with a film thickness of nominal 0.5 monolayers is shown in Fig. 3. Permalloy grows in the Volmer-Weber or island growth mode. The islands, which are typically $1 \mathrm{~nm}$ in height, are pyramid shaped and preferentially decorate the steps of the chromium crystal in addition to free standing islands on the $\mathrm{Cr}$ terraces. The base of the pyramids is oriented along the $\langle 110\rangle$ direction of $\mathrm{Cr}$. The crystallites have most likely been growing in the $\langle 100\rangle$ direction, since the interatomic distance within a nickel(001) surface is $d_{\mathrm{Ni}}=2.5 \AA$ which matches most closely the distance between two $\mathrm{Cr}$ atoms within the (001) surface of $d_{\mathrm{Cr}}=2.9 \AA$. The large lattice mismatch of about $14 \%$ leads to the island growth mode.

As spin-polarized tunneling involves mapping the local density of polarized electron states, it is important to first understand the electronic structure without regard to polarization. This way one can delineate the effects of spin polar- ization against the electronic effects of local atomic arrangement in an alloy such as NiFe. The electronic structure or equivalently the conductance $(d I / d U)$ was measured simultaneously with the topography, using a nonmagnetic tungsten tip. Figure 3(b) shows the conductance map at a bias voltage of $0.55 \mathrm{~V}$. The map reveals rich structures on the NiFe islands versus the homogeneous background of $\mathrm{Cr}$. This is a clear indication that pure $\mathrm{Cr}$ remains between the islands, and no mixing of $\mathrm{Ni}$ or $\mathrm{Fe}$ atoms has occurred on the $\mathrm{Cr}$ surface.

The plot on the right side of Fig. 3 shows normalized $d I / d U$ curves as a function of bias voltage. The black curve corresponds to clean $\mathrm{Cr}$ substrate, while the two other curves were taken above the islands marked by "1" and "2" in Fig. 3(b). The chromium spectrum shows a peak at $U_{\text {bias }}=0 \mathrm{~V}$ corresponding to the surface state at the Fermi level. The spectra 1 and 2 are very similar to the $\mathrm{NiFe}$ spectra presented in Ref. 6. Curve 2 shows a shallow peak near $U_{\text {bias }}=0.9 \mathrm{~V}$ which indicates the presence of iron in a nickel matrix. In contrast, curve 1 shows a peak near $U_{\text {bias }}=0.5 \mathrm{~V}$ which we believe indicates $\mathrm{Fe}$ within the NiFe surface. The spectroscopy reveals that despite the apparent topographical similarity of the NiFe islands, the physical placement of the $\mathrm{Fe}$ atoms was diverse. It is clear that the $\mathrm{NiFe}$ grown on $\mathrm{Cr}$ remains in alloy form rather than forming separate $\mathrm{Ni}$ and $\mathrm{Fe}$ islands. More importantly, these results point to interesting bias voltages where spin-polarized effects could be observed: the electron hole state at $0.9 \mathrm{eV}$ should show a relatively high spin polarization ${ }^{7}$ and the state near $0.55 \mathrm{eV}$, whether or not it turns out to represent $\mathrm{Fe}$ within the surface, could show interesting spin-polarized behavior.

\section{ACKNOWLEDGMENT}

This work was supported by NSF CAREER (R.D.G.) under Grant No. 9984797 and the Laboratory for Physical Sciences.

${ }^{1}$ M. Bode, Rep. Prog. Phys. 66, 523 (2003).

${ }^{2}$ M. Kleiber, M. Bode, R. Ravlic, and N. Tezuka, J. Magn. Magn. Mater. 240, 64 (2002).

${ }^{3}$ R. Ravlic, M. Bode, and R. Wiesendanger, J. Phys.: Condens. Matter 15, S2513 (2003).

${ }^{4}$ V. Maurice, S. Cadot, and P. Marcus, Surf. Sci. 458, 195 (2000).

${ }^{5}$ M. Schmid, M. Pinczolits, W. Hebenstreit, and P. Varga, Surf. Sci. 377, 1023 (1997).

${ }^{6}$ M. Dreyer, H. Pandana, and R. D. Gomez, IEEE Trans. Magn. 39, 3459 (2003).

${ }^{7}$ P. E. Mijnarends, S. Sahrakorpi, M. Lindroos, and A. Bansil, Phys. Rev. B 65, 075106 (2002). 